third factor rather than any one of these other five might not be the one to look at. This is in any case most unlikely, as in the second part of our study we looked at the four clinical features considered most commonly to be associated with endogenous depression and the four most commonly associated with neurotic depression. These showed no tendency to be distributed bimodally. However, as soon as convenient we will carry out the analysis suggested by Garside with at least the next three factors.

\section{N. McConaghy.}

School of Psychiatry,

University of New South Wales.

\section{Dear Sir,}

Two letters, one from Dr. Rosenthal (Journal, October, 1967) and one from Dr. McConaghy (see above), have attributed to one of us opinions which he does not hold. Since misunderstandings may continue to arise, we welcome the opportunity of stating our position regarding the following:

1. All depressions are either "endogenous" or "neurotic". This is not our view. We believe that other types of depression may, and probably do, exist.

2. Patients with different types of depression fall into discrete groups, with mutually exclusive symptoms. Again, this is not our view. On the contrary, many patients show both neurotic and endogenous symptoms. This does not mean, however, that depression must be a unitary disorder, that is a disorder which cannot be subdivided on the basis of aetiology or of symptoms. There are four lines of evidence that suggest that depression is not unitary:

(i) The neurotic - supposedly milder-form tends to have lasted longer and to respond less well to E.C.T.

(ii) The difficulty of including the cyclic and manic disorders in a unitary concept that embraces the neurotic varieties of depression, without sacrificing important pragmatic distinctions (treatment, prognosis). Of course, if similarities, and not differences, are being looked for, the concept could be made broad enough to include all diseases known to man.

(iii) Factor analyses have shown that the relationships between depressive symptoms cannot be explained by one general factor only. Kiloh and Garside (1963) and Carney et al. (1965) found a bipolar factor ("endogenous-neurotic") which accounted for more of the total variance than the general factor. Rosenthal and Gudeman ( $1967 a$ and b) found two factors corresponding to "endogenous pattern" and "selfpitying constellation". These two factors were orthogonal, suggesting, as McConaghy rightly points out, that the corresponding syndromes are independent, that is, uncorrelated. But this independence is consistent with the conclusions of Kiloh and Garside (1963) in their paper entitled "The independence of neurotic depression and endogenous depression". There is, therefore, no conflict between the two sets of data if "self-pitying constellation" and "neurotic" are regarded as descriptive of the same syndrome. Independence necessarily involves some overlap, and Kiloh and Garside's data should not be regarded as indicating that both symptoms and patients form two mutually exclusive groups.

(iv) The demonstration of bimodality of patients' scores. As Professor Moran (1966, p. I 168) has pointed out "the evidence for such heterogeneity rests on the bimodality of the frequency distribution of the score and not on the manner in which the latter has been found". Carney et al. (1965), for example, used scores calculated by the multiple regression technique, and Sandifer et al. (1966) used a simple summation of items chosen because of their differentiating power. In both cases unequivocally bimodal distributions were obtained, thus supporting the view that some, at least, of depressed patients can usefully be diagnosed as either endogenous or neurotic. The fact that Rosenthal ( 1967 ) did not obtain a bimodal distribution of factor scores when he rotated his factors, does not necessarily indicate that the population distribution is normal. His distribution is consistent with two hypotheses: one that the population distribution is normal and the other that it is bimodal.

3. Factors describing covarying clusters of symptoms do not define patients' groups. We agree with this point of view, as far as it goes. Yet factor scores do differentiate patients, and, moreover, if the distribution of these scores is bimodal, then different groups of patients are indicated. Such bimodal distributions of factor scores have been obtained, and thus factor analysis can in practice define patient groups, though it is not suggested that factor analysis necessarily provides the best means of so doing. But in any case factor scores provide a useful and valid means of describing patients. Rosenthal, for example, has himself shown (Rosenthal and Gudeman, 1967b) that patients who differed in respect of the independently derived characteristics "apparent precipitants", premorbid personality patterns" and certain historical data also differed in their mean scores on a factor describing an "endogenous depressive pattern". Moreover, groups of patients tentatively defined as "endogenous" or "neurotic" on the basis of their factor scores have been found to differ in long-term prognosis (Kay et al., unpublished), and it would seem to be useful, if these findings are confirmed-and for practical purposes at 
least-to distinguish patients diagnostically. This makes no assumptions about the existence of other syndromes or diagnostic groups.

Dr. Rosenthal's speculations, that factor scores on his autonomous factor I may represent the degree of development of somatic concomitants of illness, and factor scores on his self-pitying factor 2 the modification of the symptom picture by pre-existing neurotic personality features, are interesting and may be true. But this dual hypothesis is a relatively complicated one, and its confirmation will be difficult, because methods of measuring somatic changes in depression are not readily available and the assessment of premorbid personality is not a simple matter. On the other hand, symptoms are more accessible and easy to study, and the hypothesis that at least two types of depression exist, and are recognizable by their symptoms, is the more economical, more useful clinically and likely in our view to be the more fruitful in the immediate future. We do not, however, at all rule out the possibility that methods of scoring "endogenous", "neurotic" and perhaps other syndromes quantitatively (? on the basis of factor scores) will be found to be superior.

Even if symptomatically different depressions could be firmly tied to different personalities, the origins of the latter would still need to be inquired into, and aetiological issues would merely have been pushed back to an earlier stage. So the problem of how different kinds of depression arise would remain. In any case, dualistic (or pluralistic) hypotheses certainly do not rule out relationships between illness and personality or other characteristics, but symptoms appear to provide the most convenient point of departure for the study of such relationships. Dr. Rosenthal himself seems to make this point when he ends his letter by saying that definition of distinct depressive populations would make it possible to study relatively homogeneous groups (and thus to make comparisons between them), a method "which we may hope will eventually lead to a greater understanding of depression"'.

The Royal Victoria Infirmary and University of Newcastle upon Tyne, Department of Psychological Medicine. Queen Victoria Road, Newcastle upon Tyne I.

\section{Referenctes}

Garney, M. W. P., Roth, M., and Garsme, R. F. (1965) "The diagnosis of depressive syndromes and the prediction of E.C.T. response." Brit. F. Psychiat., II , 659-674.
KnOH, L. G., and Garsme, R. F. (1963). "The independence of neurotic depression and endogenous depression." Ibid., 109, 451-463.

MaConachy, N. (1967). "Neurotic and endogenous depression." (Correspondence). Ibid., 114, 120-121.

Moran, P. A. P. (1966). "The establishment of a psychiatric syndrome." Ibid., 112, $1165^{-1} 171$.

Rosenthal, S. H. (1967). "Neurotic and endogenous depressions: a sceptical view." (Correspondence). Ibid., I13, $1154-1155$.

- and Gudeman, J. E. (1967a). "The self-pitying constellation in depression." Ibid., I13, 485-489.

(1967b). "The endogenous depressive pattern: an empirical investigation." Arch. gen. Psychiat., 16, $24^{1-249}$.

Sandifer, M. G., Wilson, I. C., and Green, L. (1966). "The two-type thesis of depressive disorders." Amer. $\mathcal{F}$. Psychiat., 123, 93-97.

\section{WITGHCRAFT, PSYGHOPATHOLOGY AND HALLUGINATIONS}

Dear Sir,

A postscript with some further references might be of interest to readers of my original paper.

The Jungian description of the witch-cult as a religion based on the female principle gains support from James (1959), though the psychopathological explanation does not necessarily become more plausible. The fertility rites associated with the cult of the mother-goddess have a long history, the sexual excesses at such times are even better known.

This foumal has previously dealt with witchcraft and drugs in the late Dr. Fleming's Presidential Address (1953). The title "The Insane Root" provides the key to the fuller understanding of The Birds by Aristophanes.

The Birds has generally been treated as a lighthearted fantasy out of keeping with the other works of this stern social dramatist. For once the poet is thought to have turned his attention from the contemporary scene, Athens in the throes of bitter political unrest. The play is better understood-and loses none of its force-as a pungent satire on the leadership of the city-drug-takers and loose livers. (The Athenian Society version of the The Birds styles The Nightingale as a Courtesan.) The several references to drugs in the play, at times obscure, do not seem to have interested the classical scholarsthere is a key passage which, in line with Fleming's title, would seem to settle the issue. In answer to the question of how the travellers will become feathered the reply is,

"Don't be afraid, There's a strange root we know; Once gnaw it soundly and your wings will grow." 